\title{
Los peligros de los videojuegos y el mundo virtual en la juventud. Análisis en torno a la novela Erebos de Ursula Poznanski
}

\author{
José Alberto Sánchez Berbegal \\ Universidad de Sevilla \\ jossanber7@alum.us.es \\ https://dx.doi.org.10.12795/futhark.2018.il 3.08
}

Fecha de recepción: 2.3.2017

Fecha de aceptación: 15.05.2017

Resumen: Erebos, novela juvenil del año 2010, galardonada con numerosos premios, narra la historia de unos jóvenes que se ven atrapados en el mundo de un videojuego. Los personajes sufren cambios en sus vidas, tales como la alimentación, las relaciones personales, la salud o los resultados académicos. Esto no es más que un reflejo de lo que ocurre en la actualidad a un gran número de adolescentes. El objetivo de este estudio es determinar en qué medida está relacionado Erebos con los cambios que suceden a los personajes de la novela, así como profundizar en otras consecuencias que podrían aparecer en personas con adicción a los videojuegos. Con este fin, la pregunta de investigación es la siguiente: ¿Hasta qué punto se pueden atribuir los cambios negativos en la vida de los personajes al uso que estos hacen del videojuego? La pregunta de investigación se responde mediante el análisis exhaustivo de los aspectos de la obra, así como también mediante el empleo de estudios contrastados pertenecientes a esta temática. Una vez alcanzados los resultados, se puede concluir que la obra manifiesta los principales peligros de los videojuegos en sus personajes, causados por Erebos. Esto, muy común en los adolescentes del siglo $\mathrm{XXI}$, provoca principalmente desajustes en la salud y en el comportamiento. Por ello, un estudio sociológico y psicoanalítico de la obra podría ayudar a desentrañar más aspectos relacionados con esta temática.

Palabras clave: Videojuegos, Erebos, Riesgos.

\section{Dangers of Video Games and the Virtual World in Youth. Analysis of Ursula Poznanski's Novel Erebos}


Abstract: Erebos, young adult novel of 2010, awarded with numerous awards, narrates the story of a group of young people trapped in the world of a video game. The characters suffer changes in their lives, such as diet, personal relationships, health or academic results. This is just a reflection of what is happening to a large number of teenagers today. The objective of this research is to determine to what extent Erebos is related to the changes happening to the characters in the novel, as well as to go in depth in other consequences that could appear in video game addicts. Therefore, the research question is: To what extent can the negative changes in the lives of the characters be attributed to their use of this video game? The research question is answered through an exhaustive analysis of the aspects of the work, as well as through the use of studies on this subject. Once achieved the results, it can be concluded that the work manifests the main dangers of video games in its characters, caused by Erebos. This, very common in 21 st century adolescents, causes mainly health and behavioural imbalances. Consequently, a sociological and psychoanalytical study of the work could help to unravel more aspects related to this subject.

Key words: Video Games, Erebos, Risks.

Sumario: Introducción. I. Principales objetivos del proyecto y plan de trabajo. I.I. Objetivos generales. I.2. 0jetivos especificos. 1.3. Fases de ejecución. 2. Resultados previsibles. Conclusiones.

Erebos $^{1,2}(2010)$, publicada por la escritora austriaca Ursula Poznanski ${ }^{3}$, es una novela juvenil ${ }^{4}$ que relata los acontecimientos que suceden a un grupo de

\footnotetext{
'Existe versión de la novela en 28 idiomas. Bajo el título Erebos se encuentra en alemán, turco, español, neerlandés, checo, indonesio, portugués, croata, letón, sueco, eslovaco, estonio, rumano, catalán, italiano, noruego, húngaro, polaco y esloveno. Bajo títulos distintos (en algunas lenguas hay edición que respeta el título original y otra con otro título) se encuentra en portugués, $O$ Jogo da Morte; serbio, Ereb; croata, Bog podzemlja; checo, EREBOS - Počítačová hra, která tě sleduje; búlgaro, Epeбyc; lituano, Erebas;

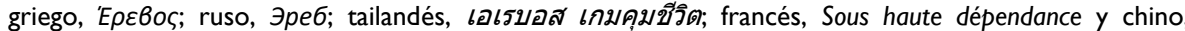
惡魔遊戲. Cabe destacar que la edición griega respeta la fonética original del título, no así el alfabeto. La información es accesible en la página web goodreads: https://www.goodreads.com/work/editions/ 0583 I70-erebos?page= I https://www.goodreads.com/work/editions/10583 I70-erebos?page=2

${ }^{2}$ Aunque en la novela dé nombre a un videojuego para ordenador, lo cierto es que Erebos, o Érebo, es un nombre relacionado con la mitología griega: "En primer lugar existió el Caos. (...) Del Caos surgieron Érebo y la negra Noche" (Muñoz 2009: II6).

${ }^{3}$ Esta escritora cuenta con una página web oficial, creada por la editorial de Erebos, Loewe Verlag: www.ursula-poznanski.de
} 
jóvenes de un instituto londinense cuando un misterioso videojuego ${ }^{5}$ para ordenador comienza a difundirse entre los alumnos de dicho centro educativo. La autora cuenta con una dilatada experiencia en el mundo de la literatura juvenil e infantil; además de Erebos, destacan en su repertorio de obras algunos títulos ${ }^{6,7}$ como Die allerbeste Prinzessin (2018), Aquila (2017) o Die Verschworenen (2013). La obra cuenta con varios premios, lo cual viene a reflejar su valía literaria y su gran acogida por parte de la crítica y los lectores ${ }^{8,9}$. En el año 2010 recibió los siguientes premios ${ }^{10}$ : Ulma Unke 2010 y Buch des Monats der Jubu-Crew Göttingen im März 2010. En 20II obtuvo: Deutscher Jugendliteraturpreis 201 I (Jugendjury)", Preis der Moerser Jugendbuch-Jury 20 $\mathrm{II}^{12}$, Endauswahl der Kalbacher Klapperschlange 201 $\mathrm{I}^{13}$, Buchfinkenpreis 2011 y Goldener Bücherwurm 20ll. A su vez, en ese mismo año consiguió dos nominaciones, Nominierung für die Goldene Leslie 2011 (Shortlist) y Nominierung für den Hansjörg-Martin-Preis 2011 .

${ }^{4}$ La literatura juvenil se podría definir de la siguiente manera: "anything that readers between the approximate ages of twelve and eighteen choose to read either for leisure reading or to fill school assignments" (Nilsen / Donelson 200I: 3).

${ }^{5} \mathrm{~m}$. Juego electrónico que se visualiza en una pantalla. http://dle.rae.es/?id=bmnbNU7

${ }^{6}$ La consulta de estos títulos está disponible en una sección de su página web oficial: http://www.ursulapoznanski.de/buecher/

7 Algunas de sus obras están también disponibles en formato de audiolibro: http://www.ursulapoznanski.de/hoerbuecher.cfm

8 “Innerhalb eines Jahres wurden 100.000 Exemplare verkauft". La información se puede encontrar en un apartado de su página web: http://www.ursula-poznanski.de/biographie.cfm

${ }^{9} \mathrm{Si}$ bien es cierto que existe una cierta controversia en torno a los premios literarios, debido a que la subjetividad tiene un papel fundamental en ellos. W. de Nooy ha llevado a cabo estudios sobre los premios literarios, en los que manifiesta: "Literary prizes occupy a central position in the literary field, since they are related to many of its institutions" (de Nooy 1989: 199). La importancia de estos premios depende de la institución que los concede y del jurado: "not all literary prizes are thought to be equally important. Some prizes are respected highly and entail a lot of publicity, whereas other prizes do not cause any commotion whatsoever. (...) The decisions and statements concerning literature of one expert are considered to be more prominent than the pronouncements of another" (de Nooy 1988: 532).

${ }^{10}$ La consulta está disponible en la página web oficial de la editorial: https://www.loewe-verlag.de/titel-00/erebos-7/09/druckansicht/7/09.pdf

II En la siguiente página web se puede encontrar información sobre este premio: http://www.djlp.jugendliteratur.org/

12 La organización que entrega estos premios cuenta con una página web oficial: https://www.moersergesellschaft.de

${ }^{13}$ Esta organización también cuenta con página web oficial: https://www.kinderverein-kalbach.de/ 
Los lectores ${ }^{14}$ atesoran un papel fundamental en la difusión de este tipo de novelas mediante la creación de blogs ${ }^{15,16}$, donde pueden manifestar de manera libre sus críticas, opiniones y/o consejos.

El desarrollo de la tecnología aumenta de manera exponencial en la actualidad. Las tereas pertenecientes a todos los ámbitos de la vida cotidiana se han colmado con herramientas novedosas, como aspiradoras que funcionan de manera autónoma ${ }^{17}$, relojes que realizan funciones mediante comandos de voz ${ }^{18} \circ$ teléfonos móviles que avisan del estado del tráfico antes de que su dueño parta hacia el trabajo. Pero sin duda, la base de todos estos recientes avances es internet ${ }^{19}$. Esta poderosa herramienta se ha convertido en el fundamento de la vida actual, pudiendo producir un colapso sin precedentes en caso de su desaparición.

Este desarrollo, como no podía ser de otra manera, ha transformado también las posibilidades de ocio de la humanidad. Los videojuegos ${ }^{20}$ han logrado un

\footnotetext{
14 “Convierten la crítica, a su vez, en un arte, paralelo y complementario” (Parra 2013: I8).

15 Por citar algunos de ellos:

http://eraseunavezpandora.blogspot.com/2018/03/erebos-ursula-poznanski.html;

http://www.kinderboekenpraatjes.nl/home-blog/erebos-upoznanski;

http://booktaught.blogspot.com/20 15/04/book-review-erebos-by-ursula-poznanski.html;

http://notodoesfantasia.blogspot.com/20 I 4/I I/erebos-un-libro-un-videojuego-la-novela.html; http://lee-hoy.blogspot.com/2012/08/resena-erebos.html
}

16 J. Sutherland manifiesta la importancia de los blogs en la difusión de los bestsellers: "Bestsellers are increasingly subjects of attention on the blogosphere, with its blogcrit, blogbuzz, and bloghype. There are new web-based discussion boards whose enthusiasms generate sales (or the opposite). Word of mouth -that most effective retail commodity- is now cyber-propelled and digitally supercharged" (Sutherland 2007: 109-110).

${ }^{17}$ Un estudio llevado a cabo en la Universidad de Estocolmo compara la función de estas máquinas con los dioses de la mitología griega y romana: "In Greek and Roman mythology the gods of metalwork could build mechanical servants made from gold. (...) However, It is only in the past few decades that robots have become a reality" (Edwards / Sörme 2018: I).

${ }^{18}$ Conocidos como Smartwatch.

${ }^{19}$ En un artículo del año 1999 se proporciona una cifra aproximada del tamaño de internet: "about 800 million pages, encompassing about 6 terabytes of text data on about 3 million servers" (Steve / Giles 1999: 107). Dicho tamaño crece de manera exponencial con el paso de los años, ya que internet va ganando cada vez una mayor relevancia.

20 Pese a que el presente estudio plantea los peligros de los videojuegos en la juventud, existen evidencias de que estos tienen notables beneficios en distintas áreas, especialmente en la enseñanza y en el desarrollo de habilidades cognitivas: "Videogames can clearly consume the attention of children and adolescents. However, it is important to assess the extent that videogame technology had an impact on childhood education. Since videogames have the capacity to engage children in learning experiences, this has led to the rise of 'edu-tainment' media. Just by watching children it becomes very clear that they 
espacio privilegiado en la oferta de entretenimiento ${ }^{21}$, en especial en el ámbito infantil y juvenil ${ }^{22}$. La obra que es objeto de análisis en este estudio gira en torno a un videojuego, Erebos. En un instituto de Londres ${ }^{23}$ comienzan a circular copias del juego. Nick, protagonista de la novela, empieza a observar comportamientos extraños en sus compañeros. Su amigo Colin deja de lado los entrenamientos de baloncesto sin previo aviso. "Sicher. Hab bloß nicht viel geschlafen letzte Nacht" (Poznanski 2014: 9) ${ }^{24}$. De esta manera replica Colin la pregunta de su amigo acerca de su salud. El abandono del deporte es una consecuencia directa provocada por los videojuegos ${ }^{25}$. Estos ofrecen al usuario una experiencia cada vez más realista, que complementa a los atributos que generan el divertimento y a la propia historia del juego. La realidad virtual ha irrumpido con fuerza en este sector, proporcionando la inmersión total en un mundo digital ${ }^{26}$. Por este motivo, un gran

prefer this type of approach to learning" (Griffiths 2002: 47). La gamificación es una de las herramientas que se emplean en la actualidad para sacar partido de los videojuegos. Esta ha sufrido un gran desarrollo en los últimos años: "Gamification meint die Übertragung von Spielelementen auf spiel-fremde Bereiche" (Hebben 2017: 19).

21 "Games are part of everyday life. Americans spent over $\$ 25$ billion on video games in 2010" (Muntean 201 I: 324).

22 Una encuesta llevada a cabo en SAPO Campus, Portugal, así lo demuestra: "youth/students are the group that presents more positive attitudes regarding the recreational use of video games" (Sousa $/$ Henriques / Costa 2017: 2339).

${ }^{23}$ La obra, escrita en lengua alemana por una escritora austriaca, se sitúa en Londres. Según expresa R. Ramos Carmona en su TFG titulado Erebos, un juego peligroso. Análisis de la estructura familiar como posible propuesta didáctica, este hecho podría no ser casualidad: "esto puede deberse a un intento de la autora por adaptarse a las novelas distópicas juveniles de tema tecnológico, que suelen situarse en un entorno anglosajón" (Ramos 2018: 8).

${ }^{24}$ Todas las referencias son de esta edición.

${ }^{25}$ El surgimiento de los esports ha supuesto una revolución en este aspecto. En la actualidad existen equipos, integrados por jugadores profesionales, que compiten en ligas de videojuegos. Estas competiciones atraen a un nutrido público y son retransmitidas en directo. En España existe una organización encargada de gestionar los esports, llamada Liga de Videojuegos Profesional (de ahora en adelante LVP). La importancia de la LVP se puede observar mediante sus patrocinios, ya que cuenta con algunos de gran calado en la sociedad como LaLiga, Banco Santander o el gigante de telefonía francés Orange. Más información acerca de esta institución se puede obtener en su página web oficial: https://www.lvp.es/

${ }^{26}$ Sin embargo, la realidad virtual supondría una disminución de la realidad: "Pourtant, à bien y réfléchir, la réalité augmentée correspond à une diminution de la réalité. Pourquoi? Parce que le réel correspond aussi et surtout - et cette fois-ci on se tourne plutôt vers Nietzsche -, au luxuriant, à l'imprévisible, au sauvage, au non-programmé et au nonprogrammatique. A l'événement au sens propre. Le réel, c'est le surgissement. Quand on ajoute de la réalité augmentée sous forme numérique, on ajoute bien évidemment du calcul, de l'anticipation, du programme, c'est-à-dire du prévisible" (Besson / Prince / Bazin 2017: 4).

Futhark I3 (20 I8) 
número de jugadores habituales ${ }^{27}$ adopta una vida sedentaria ${ }^{28}$. Abandonar el deporte para llevar a cabo otras actividades, dentro de las cuales entran los videojuegos, es la principal causa de abandono según varios estudios recopilados por K. J. Linder y otros investigadores de la Universidad de Manitoba en 199I: "the frequency of the specific reasons given for withdrawal vary (...) The most frequently encountered among these is: 'Other things to do"' (Lindner / Johns / Butcher 1991: 4). Este hecho aumenta las posibilidades de sufrir enfermedades a largo plazo. Una investigación de la Canadian Diabetes Association pone como ejemplos de comportamiento sedentario "TV viewing, seated video game playing, prolonged sitting" (Saunders / Chaput / Tremblay 2014: 53). No solo la falta de movimiento puede provocar problemas, en su mayoría cardiovasculares ${ }^{29}$, sino también la ingesta de comida, cuya calidad parece estar relacionada de manera inversamente proporcional al nivel de sedentarismo ${ }^{30}$ :

Markers of adiposity and cardiometabolic risk are positively associated with sedentary behaviours in general and with screen-based sedentary behaviours in particular. These relationships appear to be due to the influence of screenbased sedentary behaviours on food intake and may also be due to a direct metabolic impact of prolonged sitting 3 !.

El descuido de los hábitos alimenticios ciertamente afecta a Nick. Él queda con otros jugadores para pasar la noche investigando acerca de algunos aspectos de juego. Durante el transcurso de esta, una jugadora le comenta "Ich kann Muffins und Pizza durcheinanderessen" (390). Esto demuestra que no mantienen una buena dieta. Además, después aparecen más signos de la pésima alimentación: "Sie

27 "estudios realizados con niños y jóvenes españoles muestran que los jugadores habituales -que juegan más de una vez a la semana- corresponden a cifras que oscilan entre un $48 \%$ (...) y un 66\%" (Alonqueo / Rehbein 2008: 13).

28 "It has been estimated that over one-third of all participants between ten and seventeen years of age withdraw from their sport every year" (Lindner / Johns / Butcher 1991: 4).

29 Estos problemas cardiovasculares podrían verse sensiblemente agravados cuando se mantiene contacto con juegos agresivos. Un estudio de la Universidad de Bolonia comprobó los niveles de presión sistólica antes, durante y después de jugar a un juego de estas características: "The MANOVA of the physiological measurements made during the experimental session showed a significant difference between the systolic blood pressure values in the three pre-, during and post-game measurements (...), giving higher pressure values while playing compared to the other two time points" (Baldaro et al. 2004: 205).

${ }^{30}$ La relación se da de manera inversa; es decir, cuanto mayor es el nivel de sedentarismo, menor es el nivel que se le atribuye a la calidad de la comida que se ingiere.

${ }^{31}$ Ibíd. 
winkten ab, nahmen den Pizzarest und öffneten eine Dose Erdnüsse" ${ }^{\prime 2}$. Esto podría agravar algunos problemas previos que el protagonista pudiera tener, ya que parece que su situación doméstica previa al descubrimiento de Erebos no era especialmente adecuada. Esto se puede comprobar en la lista de la compra que toma Nick antes de ir al supermercado: "Tiefkühlpizza ohne Ende, außerdem Lasagne, Fischstäbchen und Fertignudelgerichte" (25). "Heute gibt's nur Hotdogs" (7) afirma su madre en otra ocasión; mantener una alimentación como esta de manera continuada puede provocar carencias, las cuales ella parece compensar mediante la toma de suplementos vitamínicos: "Nick ließ seine Sporttasche in die Ecke fallen und warf eine Aspirin plus C-Tablette in ein Glas mit Wasser" ${ }^{\text {"33,34. }}$

Nick recibe una copia del juego de manos de una compañera de clase. Todo transcurre con cierto tono reservado y misterioso, siguiendo las reglas que marca el propio juego a sus usuarios: "Du darfst es niemandem zeigen. Sonst funktioniert es nicht. Du musst es gleich einstecken und sag keinem, dass ich es dir gegeben habe. Versprochen?" (28). De esta manera, ella trata de asegurarse de que el protagonista sigue las normas del juego. El chico instala Erebos en su ordenador y queda sorprendido con el contenido de este, afirmando "Das sieht echt aus, so echt" (38). Los personajes del juego mantienen conversaciones muy realistas ${ }^{35}$, impropias de la inteligencia artificial ${ }^{36}$ del momento ${ }^{37,38}$. Más adelante, el juego le

\footnotetext{
32 Ibíd.

${ }^{33}$ Ibíd.

${ }^{34}$ Existen numerosos estudios que demustran las propiedades de la vitamina $C$, especialmente su papel como antioxidante: "A number of studies in the human have attempted to demonstrate the effects of vitamin $C$ on vascular responsiveness, intestinal iron absorption and reduction of harmful oxidants in the stomach. (...) Vitamin $C$ and the other antioxidant vitamin, vitamin $E$, appear to have beneficial effects on vascular endothelial function in healthy subjects and in patients with cardiovascular disease" (Padayatty et al. 2003: 25).

${ }^{35}$ La disciplina que se encarga de hacer posible la comunicación entre los seres humanos y las máquinas mediante el lenguaje natural, tal y como lo hace Nick, es la lingüistica computacional: "Die computerlinguistik ist das Fachgebiet, das sich mit der maschinellen Verarbeitung natürlicher Sprache beschäftigt" (Schmitz 2013: I).

36 "In der Informatik bezeichnet KI eine Richtung der Forschung, die versucht, menschliches intelligentes Verhalten durch einen Algorithmus zu imitieren” (Rath 20I7: 8). En la cita, KI hace referencia a künstliche Intelligenz.

37 "Die künstliche Intelligenz im Jugendbuch Erebos erreicht das von Alan M. Turing prognostizierte Leistungsvermögen. Sie ist in der Lage mit den Mitteln der Sprache den Spieler*innen zu suggerieren eine Person zu sein" (Herr 2017: 16).

${ }^{38}$ La inteligencia artificial presente en Erebos podría superar el Test de Turing. Nick, cuando afirma "Das sieht echt aus, so echt" (38), determina que el videojuego parece ser real, ya que constantemente, $\begin{array}{ll}\text { Futhark I3 (2018) } & 125\end{array}$ 
pide introducir su nombre. Tras tres intentos fallidos de introducir uno falso, finalmente acaba escribiendo el verdadero, tras lo cual el chico puede continuar. Nick siente una sensación de miedo, ya que Erebos parece ser más que un programa de ordenador y saber más de lo que debería ${ }^{39}$ :

Das Gefühl, beobachtet zu werden, war beängstigend, und Nick tastete nach den Kopfhörern, um sie sich von den Ohren zu ziehen. Doch die Schrift verschwand bereits, ebenso die Stimmen, und eine lockende Melodie setzte ein, ein versprechen von Geheimnis und Abenteuer (45-46).

Uno de los peligros de internet y los videojuegos online es el uso fraudulento que se puede hacer de las posibilidades de interacción entre usuarios ${ }^{40}$. Es frecuente descubrir que un adulto ha conseguido informaciones 0 materiales privados $y$, en ocasiones, también sensibles de un joven mediante engaños o extorsiones. Esto se conoce como ciberacoso o cyberbullying ${ }^{41}$. El miedo que siente el protagonista cuando se percata de que el juego parece saber muchas cosas se puede relacionar con el miedo que sienten estas víctimas cuando el acto de extorsión se ha consumado y el menoscabo es notable. Estos sucesos provocan desenlaces que en muchos casos pueden resultar fatales. Principalmente, se puede afirmar que: "contributes to depression, decreased self-worth, hopelessness, and loneliness - all of which are precursors to suicidal thoughts and behavior" (Hinduja / Patchin 2010: 207).

A continuación, Nick recibe algunas reglas que debe cumplir para poder seguir jugando:

\footnotetext{
debido a las variadas conversaciones que puede mantener con un personaje, tiene la sensación de estar frente a algo más que un software, a una inteligencia superior. El Test de Turing consiste en dejar que un sujeto seleccionado para el experimento mantenga una conversación. Tras esta, la persona debe manifestar si ha mantenido la conversación con una persona o con una máquina.

39 El gran potencial de Erebos tiene relación con el desarrollo exponencial de la tecnología: "A contemporary issue has arisen that given its exponential growth, technology could be a potential sole successor to human intelligence" (Swan 20I0: 15I). Según M. Swan, la tecnología podría llegar a ser la sucesora de la inteligencia humana. Por tanto, cuando Nick se sorprende por la gran inteligencia que muestra el juego, no está más que experimentando este cambio causado por el veloz desarrollo de la tecnología.

${ }^{40}$ H. Cole y M. D. Griffiths aportan datos sobre la interacción online entre jugadores. En este caso, se puede saber el porcentaje de personas que afirman haber hecho buenos amigos en el juego: "Approximately three quarters of both males (...) and females (...) said they had made good friends within the game" (Cole / Griffiths 2007: 577).

${ }^{4 I}$ Se puede definir como: "bullying through e-mail, instant messaging (IM), in a chat room, on a Web site, on an online gaming site or through digital messages or images sent to a cellular phone" (Kowalski et al. 2012: 1).
} 
Du hast nur eine Chance, Erebos zu spielen. Wenn du sie vertust, ist es vorbei. Wenn deine Figur stirbt, ist es vorbei. Wenn du gegen die Regeln verstößt, ist es vorbei. (...) Wenn du spielst, achte darauf, allein zu sein. Erwähne niemals im Spiel deinen richtigen Namen. Erwähne niemals außerhalb des Spiels den Namen deines Spielcharakters. (...) Der Inhalt des Spiels ist geheim. Sprich mit keinem darüber. (...) Bewahre die Erebos-DVD sicher auf. (...) Kopiere sie auf keinen Fall, außer der Bote fordert dich dazu auf (46-47).

Algunas de estas reglas, como el hecho de solo tener una oportunidad para jugar, deber encontrarse en solitario, mantener el juego en secreto o no proporcionar copias sin haber recibido un requerimiento previo por parte del propio juego, conforman un cierto misterio en torno a Erebos. El matiz se torna más enigmático cuando el videojuego pide a Nick que realice tareas en la vida real $^{42}$. "Das freut mich. Warte nicht zu lange. Wir sehen uns morgen, noch vor dem Mittag. Bis dahin muss deine aufgabe erfüllt sein. Falls du mich enttäuschst..." (8I). De esta manera comunica el mensajero del juego al jugador cuándo debe cumplir la tarea, condición sine qua non para poder continuar con la aventura del videojuego. Tanto las minuciosas reglas, como el requisito de cumplir una labor en la vida real, se pueden relacionar con la extorsión anteriormente mencionada que sufren algunos jóvenes en internet. Una persona oculta bajo una identidad digital, en este caso representada por Erebos, requiere determinadas acciones a su objetivo, así como también le marca de manera exhaustiva los pasos a seguir. En ocasiones, como también sucede en la novela, los requerimientos van subiendo su complejidad y severidad de manera paulatina, pudiendo llegar al intercambio de fotos privadas, el daño físico o el suicidio. En la novela, Erebos acaba pidiendo un asesinato; esto se puede relacionar con un fenómeno ocurrido en 2016 en las redes sociales que causó muertes en todo el mundo, Blue Whale Challenge ${ }^{43}$ :

Blue Whale Challenge is a suicide game that was released in Russia in 2013 and includes a series of tasks that has to be completed over a period of 50 days and with each passing day the tasks become more and more dangerous and life-threatening. (...) The inventor's purpose is to 'clean the society of biological

\footnotetext{
${ }^{42}$ G. Miller publicó un artículo en 2007 en la prestigiosa revista Science. En él incorpora una afirmación de dos investigadores de la Universidad Cornell de Nueva York: "Other researchers have also seen evidence of a carryover from the virtual world to the physical one" (Miller 2007: 1342). Esta aseveración se basa en un estudio llevado a cabo por ellos mismos, en el que descubren que un personaje situado en un mundo virtual puede influir en la visión que tiene esa persona de sí misma en la vida exterior al juego: "we're seeing that they're actually thinking about themselves differently" (Miller 2007: 1342). Esto aparece en la novela de Ursula Poznanski que trata este estudio. Erebos es un videojuego que pide a sus usuarios que realicen tareas en la vida real; es decir, en cierta manera lleva al personaje virtual fuera del software y lo introduce en el jugador, de manera que este vive la vida real influenciado por el juego.

${ }^{43}$ Conocido en español como Reto de la ballena azul.
} 
wastes' as he intended to 'clean' the society from individuals considered as a burden on the society (Mukhra et al. 2017: 2) ${ }^{44}$.

Este reto, perpetrado a través de las redes sociales, conseguía que algunos jóvenes pusieran fin a su vida, siguiendo las directrices de una persona desconocida.

El protagonista, dubitativo por la orden que acaba de recibir, siente ansiedad al comprobar que el juego no funcionará hasta que cumpla con su cometido. Pasada la media noche no consigue dormir. Comienza a jugar al Buscaminas y se plantea navegar por internet. La falta de sueño que puede llegar a provocar la adicción a un videojuego en un joven es otro aspecto de relevancia en torno al tema del estudio. En la obra, Colin comenta a su amigo que duerme poco. Esta falta de sueño, como finalmente ocurre con este personaje, puede conducir a un bajo rendimiento académico. Un estudio realizado en un grupo de 592 estudiantes adolescentes afirma que: "los sujetos que presentan un patrón de sueño medio, obtienen mejores notas (...) que los de patrón corto" (Quevedo-Blasco / Quevedo-Blasco 20II: $5 \mathrm{I})^{45}$. Colin comienza a faltar a las clases. Al igual que ocurre con él al principio de la obra, sucede con Nick cuando recibe ese primer encargo de Erebos. A partir de ese momento, debido a su completa inmersión dentro de los quehaceres del juego, comienza a abstraerse de lo que ocurre a su alrededor:

Es fiel Nick schwer, sich auf die Worte seiner Mutter zu konzentrieren. Sarius hatte vorhin ein Zimmer in einer Schänke der Weißen Stadt bezogen und der dort zuständige Wirt hatte ihn drei Stunden Ruhe verordnet $(118)^{46}$.

El juego marca los tiempos, algo que crea ansiedad en el jugador ${ }^{47}$. En la actualidad abunda la presencia de este tipo de juegos en las tiendas de aplicaciones de los dispositivos móviles. Juegos en los que cada cierto tiempo es necesario realizar una serie de tareas que permiten ir mejorando un personaje de manera

\footnotetext{
44 Aquí se pueden consultar las cincuenta tareas que componen el reto: http://makeubig.com/20I7/09/20/blue-whale-challenge-list-50-tasks/

45 En el estudio se establece que el sueño medio comprende un rango de siete a nueve horas, mientras que el sueño corto se prolonga durante un tiempo inferior a siete horas.

${ }^{46}$ Sarius es el nombre que recibe el personaje de Nick dentro del juego.

${ }^{47}$ La impaciencia que siente Nick en esta situación es un signo de adicción a la tecnología; en esta ocasión a los videojuegos. Un estudio llevado a cabo en la Universidad Ramón Llull de Barcelona muestra tres síntomas comunes a las personas con este tipo de adicción, los cuales se manifiestan de igual manera en los adictos a alguna sustancia: "Psychological dependence. Includes desire, anxious impatience, craving and attentional polarization or focalization (the activity takes on paramount importance, dominating thoughts and feelings)" (Castellana et al. 2007: 197). Esta cita muestra el segundo síntoma establecido por el estudio, en el que se menciona la aparición de ansiedad e impaciencia.
} 
gradual $^{48}$. Las notificaciones que envían al usuario invaden su vida personal y provocan que éste esté persistentemente pensando en las labores que debe realizar en su pequeño mundo virtual. Un estudio presentado en la revista Cyberpsychology, Behavior and Social Networking en el año 2010 demuestra que este tipo de juegos favorecen la aparición de una ansiedad moderada: "Mean scores for trait and state anxiety were 50 and 52.1 (of 80) respectively. This indicated that participants felt generally anxious" (Mehroof / Griffiths 2010: 315).

Asimismo, el protagonista de la novela empieza a descuidar los aspectos relacionados con sus estudios, al igual que le sucedió a su amigo Colin con anterioridad:

Noch 43 Minuten. Er rieb sich das Gesicht mit beiden Händen, bevor er sich wieder über sein Buch beugte und auf die Formeln starrte. Wenn er wenigstens ein paar erste Sätze für seine Arbeit fand, war das für heute genug. (...) Obwohl, auf diese Weise lohnte das Arbeiten sich nicht. Wenn schon schreiben, dann richtig. Sich viel Zeit nehmen, am besten morgen, nach dem Frühstück (I2I).

Comienza a retrasar la escritura de un trabajo de química mediante excusas, quedando este en un segundo plano.

Das Computerspiel übt eine große Faszination auf die Spieler*innen aus, deshalb vernachlässigen sie ihr Privatleben und die Schule. Außerdem sind einige Spieler*innen bereit alles dafür zu tun, um im Computerspiel erfolgreich zu sein (Herr 2017: 15).

La adicción que provoca Erebos es causante del descuido del ámbito académico. Así lo demuestra una investigación llevada a cabo en el año 2012 en el sur de España con la participación voluntaria de un grupo de escolares. Los sujetos con adicción a videojuegos reciben la siguiente descripción: "these players show higher absenteeism, lower satisfaction with their school and teachers, worse relationships with their teachers" (Tejeiro et al. 20I2: 3/2). Una alta tasa de absentismo y malas relaciones con el profesorado, todo basado en la baja satisfacción. No es de extrañar que en la novela se relate la presencia de la policía en el instituto, motivada por el robo de algunos ordenadores: "Die allgemeine Aufmerksamkeit galt zwei Polizisten, die zielstrebig auf den Korridor zugingen, der zur Direktion führte. (...) Neun Computer sind geklaut worden, nagelneue Notebooks" (25-26). Es posible que el robo sea consecuencia de los aspectos anteriores relacionados con las consecuencias de la adicción a los videojuegos ${ }^{49}$.

\footnotetext{
${ }^{48}$ Por nombrar algunos de los actuales: Clash of Clans, Pokemon Go, Clash Royale.

${ }^{49}$ Los jóvenes con adicción a los videojuegos tienden a adoptar comportamientos ilegales, además de mostrar menos consideración hacia los demás. Así lo demuestran los resultados de un estudio de 2012 publicado en la revista Psychology: "they score higher in introversion, hardness and tendency to anti-

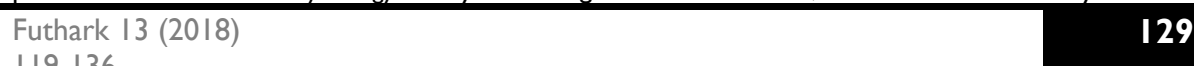


La adolescencia es el periodo previo a la edad adulta, en el cual los jóvenes sufren cambios en sus gustos, comportamientos y amistades ${ }^{50}$. Si la adicción a un videojuego acapara las motivaciones de estos cambios, en el futuro aparecerán problemas $^{51}$, puesto que es conocido que: "friendships at these ages have a major influence on the development of personality, social skills and social behavior" (Berndt 1982: 1447). Las amistades de Colin, para sorpresa de Nick, cambian; su amigo empieza a compartir demasiado tiempo con Dan y Alex, a quienes ellos no tenían aprecio. "Dan und Alex? Nick blinzelte ungläubig. Die beiden waren so uncool, dass Colin sie immer nur >die Häkelschwestern vor, sich bei den beiden als Nummer 3 zu bewerben?" (9). De esta manera muestra el protagonista su sorpresa al ver el cambio de amistades. De igual manera, Nick comienza a modificar su comportamiento en el ámbito social. Al recibir una llamada de su amigo Jamie, rechaza la posibilidad de quedar con él mediante una serie de mentiras: "Ist echt eine gute Idee, aber ich sitze gerade an meiner ChemieHausarbeit" (I23). Cuando Jamie se ofrece para ayudarlo, de nuevo se sirve de una excusa para eludir la proposición: "wahrscheinlich kann ich mich alleine besser konzentrieren" 52 . Finalmente, finaliza la llamada con otra excusa, debido a que el tiempo de espera para poder jugar de nuevo había llegado a su fin: "Hör mal, ich muss auflegen. Mum ruft gerade nach mir" (124). El rechazo de la vida exterior con la finalidad de mantenerse dentro de un mundo virtual es frecuente en los casos de adicción a los videojuegos. Un artículo publicado en 1983 en Journal of Youth and Adolescence muestra el siguiente resultado en relación a las consecuencias de la soledad ${ }^{53}$ en los adolescentes:

These results reveal that the lonelier the adolescent, the more likely he or she was to be anxious, depressed, show an external locus of control, have high levels of public self-consciousness and social anxiety, and exhibit low levels of happiness and life-satisfaction. In addition, loneliness was associated with a reluctance to take social risks (Moore / Schultz 1983: 99).

social and illegal behaviors, and they score lower in sincerity, empathy, consideration for the other and self-concept" (Tejeiro et al. 20I2: 3/2).

${ }^{50}$ Few developmental periods are characterized by so many changes at so many different levels" (Eccles 1993: 90)

${ }^{51}$ T. J. Berndt, investigador de la Universidad de Yale, asegura: "special importance is attributed to friendships during early adolescence, that is, during the period from roughly 12 to 16 years of age" (Berndt 1982: 1447).

52 lbíd.

53 "Ioneliness is a response to a relational deficit that gives rise to a yearning for the insufficient relationship" (Rotenberg / Hymel 1999: II). 
Ansiedad, depresión ${ }^{54} \circ$ bajos niveles de felicidad pueden ser los síntomas que aparezcan en los jóvenes que se encuentran en esta circunstancia. Sin embargo, la realidad del juego no sería más que una ausencia, tal y como afirma S. Nesteriuk:

O jogador é, desta forma, também enredado pelas própias formas expressivas do jogo e da narratividade no videogame, que assim como a reticularidade cotidiana, o envolvem em uma presença feita de ausência, na qual o jogo e a narrativa se apresentam senhores de seus próprios cronotopos (Nesteriuk 2004: 10). la realidad.

El jugador se ve atrapado por los cronotopos ${ }^{55}$ del juego, que lo apartan de

\section{Erebos se emplea como material didáctico:}

La línea didáctica habitual se centra en la adicción que los juegos de ordenador o videojuegos pueden llegar a producir entre los jóvenes, además de la incapacidad de sus usuarios de distinguir la ficción de la realidad. (...) según la web del Gymnasium-Ohmoor (Hamburgo), en el año 2015 se realizó la adaptación teatral de Erebos por parte de los alumnos del grupo Lebendige Kultur (...) La actividad llevada a cabo demuestra claramente que la orientación de análisis que se sigue es la adicción a los videojuegos y la pérdida, por parte de los jugadores, de la línea que separa la realidad de la ficción (Ramos 2018: 4).

El jugador sufre una inmersión en un mundo paralelo ${ }^{56}$ que le proporciona la visión y la experiencia de otra realidad. Cuando la realidad del mundo virtual es más atractiva ${ }^{57}$ que la propia vida del individuo, comienzan a aparecer las dificultades.

No videogame experimentamos a imersão em ambientes horizônticos, a possibilidade da compreensão que se dá pela própria experiência do jogador diante de uma realidade sempre maior e independente, que é construída e

\footnotetext{
${ }^{54}$ La depresión puede ayudar a que aparezca el absentismo escolar, según un estudio de la Universidad de Gante: "Depressieve klachten kunnen leiden tot absenteïsme op school" (Braet / Bijttebier / Bosmans 2017: 161$)$.

55 "In the literary artistic chronotope, spatial and temporal indicators are fused into one carefully thought-out, concrete whole. Time, as it were, thickens, takes on flesh, becomes artistically visible; likewise, space becomes charged and responsive to the movements of time, plot and history. The intersection of axes and fusion of indicators characterizes the artistic chronotope" (Bakhtin 1981: 84).

${ }^{56}$ Diferente del mundo real.

57 "los videojuegos (...) a causa de su carácter lúdico, constituyen una experiencia altamente atractiva y motivadora para los jugadores” (Alonqueo / Rehbein 2008: I3).
} 
reconstruída pela própia ação do jogador por meio da interatividade proporcionada pela hipermídia (Nesteriuk 2003: s.p.).

Siguiendo la afirmación anterior, el protagonista de la novela se ve inmerso en el mundo que le ofrece el juego. Esta inmersión se desarrolla dentro de una presión social reinante en el entorno del centro educativo al que acude el chico. La presión que realiza un colectivo sobre una persona se conoce con el término anglosajón peer pressure ${ }^{58}$. D. R. Clasen y B. B. Brown definen el término como "pressure to think or behave along certain peer-prescribed guidelines" (Clasen / Brown 1985: 452). En un momento determinado, un gran número de alumnos comienza a centrar su existencia en el juego. Tras esto, Nick siente la necesidad de saber en qué consiste aquello que tanto éxito está teniendo entre sus compañeros y no duda en adentrarse en el mundo de Erebos. Este hecho es habitual, ya que es algo primario, forma parte de la naturaleza humana: "Peer pressure is a primary mechanism of transmitting group norms and mantaining loyalties among group members" 59 .

Una vez expuestos los peligros que entrañan los videojuegos y el mundo virtual y la manera en la que se manifiestan en Erebos, cabe concluir que la obra, con independencia de una posible voluntad de la autora por reflejar estos hechos ${ }^{60}$, manifiesta de manera clara dichos peligros a través de los personajes y sus acciones. La juventud, especialmente la adolescencia, es una etapa de constantes cambios; el paso de la infancia a la edad adulta marca la importancia de esta etapa. Por tanto, el desarrollo del individuo se torna de vital trascendencia durante este periodo de su existencia. Los videojuegos, algo presente en la vida cotidiana actual, tienen la capacidad de influir en un sujeto, tanto de manera positiva como de manera negativa. Es por eso por lo que se antoja necesario un control del uso que se hace de ellos; de lo contrario, los jóvenes podrían comenzar a sufrir las nocivas consecuencias de estos medios digitales -tales como el impulso hacia lo ilegal, la mala alimentación, el sedentarismo o el absentismo escolar. Sin duda, un análisis sociológico y psicoanalítico de la obra podría ayudar a profundizar más en los aspectos expuestos en el presente estudio y permitiría desentrañar particularidades

\footnotetext{
58 "young people perceive peer pressure to participate in substance use in order to be accepted by the peer group" (Hansen / Graham I991: 4I5). Esta afirmación va dirigida a las personas con adicción a una sustancia. En este caso, como los síntomas de la adicción a los videojuegos se asemejan a los síntomas de las adicciones a sustancias (vid. nota 42), se puede dar por válido que la presión (peer pressure) que sobre ambos grupos incide también se puede unificar.

59 Ibíd.

${ }^{60}$ No es conocido si Ursula Poznanski tuvo en cuenta todos los aspectos negativos de los videojuegos que aparecen reflejados en la obra en el momento de su escritura.
} 
intrínsecas a los personajes, de manera que se pudiera manifestar cómo sus personalidades se ven afectadas.

\section{Referencias bibliográficas}

-Bibliografía primaria

POZNANSKI, U. (20|4). Erebos. Bindlach: Loewe Verlag.

-Bibliografía secundaria

AlongueO, P.; ReHBein, L. (2008). Usuarios habituales de videojuegos: una aproximación inicial. Última Década I6 (29): II-27.

BAKHTIN, M. M. (198I). The Dialogic of Imagination: Four Essays by MM. Bakhtin. (C. Emerson \& M. Holquist, Trans.). Austin: University of Texas Press.

Baldaro, B. et al. (2004). Aggressive and non-Violent Videogames: Short-Term Psychological and Cardiovascular Effects on Habitual Players. Stress and Health: Journal of the International Society for the Investigation of Stress 20 (4): 203-208.

BERNDT, T. J. (1982). The Features and Effects of Friendship in Early Adolescence. Child Development 53 (6): I447-I460.

Besson, A.; Prince, N.; Bazin, L. (20I7). De la Pluralité des mondes: le paradigme de l'immersion dans les fictions contemporaines. Publije, e-revue de critique littéraire I: I-7.

Braet, C.; Bijttebier, P.; Bosmans, G. (20/7). De diagnostiek van depressieve klachten bij kinderen en jongeren. Diagnostiek bij kinderen, jongeren \& gezinnen 4: |6|-|72.

CastellanA, M. et al. (2007). Adolescents and Information and Communications Technologies: Internet, Mobile Phone and Videogames. Papeles del Psicólogo 28 (3): 196-204.

COLE, H. / Griffiths, M. D. (2007). Social Interactions in Massively Multiplayer Online Role-Playing Gamers CyberPsychology \& Behavior 10 (4): 575-583.

DE NOOY, W. (1988). Gentlemen of the Jury...: The Features of Experts Awarding Literary Prizes. Poetics 17: 531-545.

(1989). Literary Prizes: Their Role in the Making of Children's Literature. Poetics 18: 199-213.

ECCLES, J. S. (1993). Development During Adolescence: The Impact of StageEnvironment Fit on Young Adolescents' Experiences in Schools and in Families. American Psychologist 48 (2): 90-101.

EdWARDS, T.; SÖRME, J. (2018). A Comparison of Path Planning Algorithms for Robotic Vacuum Cleaners (Degree project in technology, first cycle). Stockholm: KTH Vetenskap och Konst. 
GRIFFITHS, M. (2002). The educational benefits of videogames. Education and health, 20 (3): 47-5I.

Hansen, W. B.; GrahaM, J. W. (I99I). Preventing Alcohol, Marijuana, and Cigarette Use among Adolescents: Peer Preasure Resistance Training versus Establishing Conservative Norms. Preventive Medicine 20: 4I4-430.

HebBEN, K. (20I7). ,Mehr als Spiele' - Optimierungsideologien des Spiels. DoLiMette 7: $17-24$.

HERR, M. (20I7). Künstliche Intelligenz im sanktionierten Jugendbuch am Beispiel ,Erebos' von Ursula Poznanski. DoLiMette 7: 8-I2.

Hinduja, S.; Patchin, J. W. (20I0). Bullying, Cyberbullying and Suicide. Archives of Suicide Research I4 (3): 206-22I.

KOWALSKI, R. M. et al. (20I2). Cyberbullying: Bullying in the Digital Age. Hoboken: John Wiley \& Sons.

LINDNER, K. J.; Johns, D. P. / Butcher, J. (199I). Factors in Withdrawal From Youth Sport: A Proposed Model. Journal of Sport Behavior I4 (I): 3-I8.

Mehroof, M.; GriffithS, M. D. (20I0). Online Gaming Addiction: The Role of Sensation Seeking, Self-Control, Neuroticism, Aggression, State Anxiety, and Trait Anxiety. Cyberpsychology, Behavior and Social Networking 13 (3): $313-316$

Miller, G. (2007). The Promise of Parallel Universes. Science 317 (5843): I34I1343.

MOORE, D.; Schultz, N. R. (1983). Loneliness at Adolescence: Correlates, Attributions, and Coping. Journal of Youth and Adolescence I2 (2): 95-100.

MukhrA, R. et al. (2017). 'Blue Whale Challenge': A Game or Crime?. Science and Engineering ethics: I-7.

MunteAN, C. L. (20I I). Raising engagement in e-learning through gamification. Proc. 6th International Conference on Virtual Learning ICVL, I, 323-329.

Muñoz, F. A. (2009). Caos, Gea y Eros. Desde el desorden a la armonía de la Paz. Convergencia. Revista de Ciencias Sociales 16: II5-140.

NesteriuK, S. (2003). Ludologia e $(\mathrm{m})$ Videogame. Sociedade Brasileira de Estudos Interdisciplinares da Comunicação. XXVI Congresso Brasileiro de Ciências da Comunicação, I-14.

NeSTERIUK, S. (2004). Breves considerações acerca do videogame. XXVIII Intercom, $\mathrm{I}-\mathrm{I} 4$.

Nilsen, A. P.; DONelson, K. L. (200I). Literature for Today's Young Adults. New York: Longman.

PAdAyATtY, S. J. et al. (2003). Vitamin C as an Antioxidant: Evaluation of Its Role in Disease Prevention. Journal of the American College of Nutrition, 22, I, I8-35.

PARRA, E. (20I3). Destrivializando lo trivial. Reflexiones en torno a la necesidad de una nueva orientación crítico-literaria, Trivialidades literarias. Reflexiones en torno a la literatura de entretenimiento, 9-24. 
POZNANSKI, U. (20I3), Die Verschworenen. Bindlach: Loewe Verlag. (2017), Aquila. Bindlach: Loewe Verlag. (2018), Die allerbeste Prinzessin. Bindlach: Loewe Verlag.

QueVEdo-Blasco, V. J.; QueVedo-Blasco, R. (20II). Influencia del grado de somnolencia, cantidad y calidad de sueño sobre el rendimiento académico en adolescentes. International Journal of Clinical and Health Psychology, I I, I, 49-65.

RAMOS, R. (20I8), Erebos, un juego peligroso. Análisis de la estructura familiar como posible propuesta didáctica (Trabajo de fin de grado). Sevilla: Universidad de Sevilla.

RATH, M. (20I7). Künstliche Intelligenz - Eine Warnung. DoLiMette, 7, 8-I 2.

RotenberG, K. J.; Hymel, S. (1999), Loneliness in Childhood and Adolescence. Cambridge: Cambridge University Press.

SAunders, T. J.; Chaput, J.-P.; TRemblay, M. S. (20l4), "Sedentary Behaviour as an Emerging Risk Factor for Cardiometabolic Diseases in Children and Youth", Canadian Journal of Diabetes, 38, 53-6I.

SCHMITZ, U. (20I3). Computerlinguistik: eine Einführung. Berlín: Springer-Verlag.

SOUSA, C.; HenriQues, S.; CostA, C. (20I7). Are videogames a waste of time? The pedagogical value of videogames: A multi-stakeholder approach. EDULEARN I7: 2336-234I.

STEVE, L.; GILES, C. L. (1999). Accessibility of information on the web. Nature 400 , 6740: 107-109.

SUTHERLAND, J. (2007). Bestsellers: a very short introduction. Oxford: Oxford University Press.

SWAN, M. (20I0). Engineering Life into Technology: the Application of Complexity Theory to a Potential Phase Transition in Intelligence. Symmetry 2: 150183.

TEJEIRO, R. A. et al. (20I2). Risk Factors Associated with the Abuse of Video Games in Adolescents. Psychology, 3 (4): $310-314$.

-Webgrafía

http://dle.rae.es/?id=bmnbNU7

http://eraseunavezpandora.blogspot.com/2018/03/erebos-ursulapoznanski.html

http://lee-hoy.blogspot.com/2012/08/resena-erebos.html

http://makeubig.com/2017/09/20/blue-whale-challenge-list-50-tasks/

http://notodoesfantasia.blogspot.com/2014/ I //erebos-un-libro-un-

videojuego-la-novela.html

http://www.djlp.jugendliteratur.org/ 
http://www.kinderboekenpraatjes.nl/home-blog/erebos-upoznanski http://booktaught.blogspot.com/2015/04/book-review-erebos-byursulapoznanski.html

http://www.ursula-poznanski.de/biographie.cfm

http://www.ursula-poznanski.de/buecher/

http://www.ursula-poznanski.de/hoerbuecher.cfm

https://clashroyale.com/es/

https://gymnasium-ohmoor.de/

https://supercell.com/en/games/clashofclans/

https://www.goodreads.com/work/editions//0583 I70-erebos?page= I

https://www.goodreads.com/work/editions//0583/70-erebos?.page $=2$

https://www.kinderverein-kalbach.de/

https://www.loewe-verlag.de/titel-0-0/erebos-7109/druckansicht/7109.pdf

https://www.lvp.es/

https://www.moersergesellschaft.de

https://www.pokemongo.com/es-es/

www.ursula-poznanski.de/ 